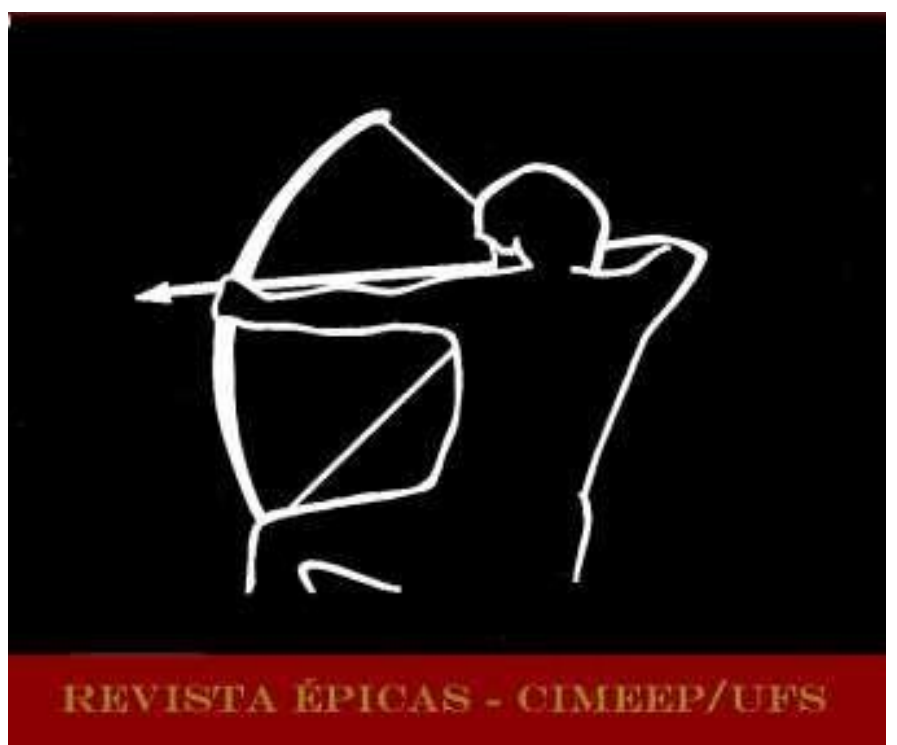

AKBARPOURAN, Monire. La semaine, o épico bíblico sobre a criação. Cordel épico. In: Revista Épicas. Ano 5, N. 10, Dez 21, p. 45-63. ISSN 2527-080-X.

DOI: http://dx.doi.org/10.47044/2527-080X.2021v10.4563

\title{
L'ESPACE ORDONNE DE LA CEREMONIE POUR CONJURER L'ESPACE ANGOISSANT DES CONQUETES : L'IMAGINAIRE DES TRADITIONS EPIQUES TURCOMANES (LE LIVRE DE DEDE KORKUT, LE LIVRE DE DEDE KORKUT QADJAR ET LES DESTAN IRANIENS DE KOROGLU) ${ }^{1}$
}

\author{
O ESPAÇO ORDENADO DA CERIMÔNIA PARA EVITAR O ESPAÇO ANGUSTIANTE DAS CONQUISTAS: \\ O IMAGINÁRIO DAS TRADIÇÕES ÉPICAS TURCOMANAS (LE LIVRE DE DEDE KORKUT, LE LIVRE DE DEDE \\ KORKUT QADJAR E OS DESTANS IRANIANOS DE KOROGLU)
}

Résumé: Les traditions épiques des Turcs Oghuz ont évolué au fil du temps et des conquêtes accomplies à partir du Kazakhstan vers l'ouest et vers le sud. Le Livre de Dede Korkut, Le Livre de Dede Korkut qadjar et les destan iraniens de Koroğlu, aujourd'hui encore récités en Iran enregistrent ces déplacements en reconfigurant les oppositions au Turc Oghuz : chrétiens en Anatolie aux $\mathrm{XV}^{\mathrm{e}}-\mathrm{XVI}^{\mathrm{e}}$ siècles, Persans en Iran deux siècles plus tard. La ritualisation très rigoureuse de l'espace de la performance lors des récitations traditionnelles contemporaines de Koroğlu permet en même temps de repérer dans l'épopée un effort pour conjurer l'angoisse de l'acculturation née des conquêtes turcomanes en Anatolie et au Caucase.

Mots clés: Le Livre de Dede Korkut. Le Livre de Dede Korkut qadjar. Les destan iraniens de Koroğlu. Performance.

Abstract: The epic traditions of the Oghuz Turks evolved over time and with the conquests accomplished from Kazakhstan to the west and south. The Book of Dede Korkut, The Book of Dede Korkut Qadjar and the Iranian destan of Koroğlu, still recited in Iran today, record these shifts by reconfiguring the oppositions to the Oghuz Turk: Christians in Anatolia in the 15th-16th centuries, Persians in Iran two centuries later. The very rigorous ritualisation of the performance space during contemporary traditional recitations of Koroğlu allows at the same time to identify in the epic an effort to ward off the anguish of acculturation born of the Turkic conquests in Anatolia and the Caucasus. Keywords: The Book of Dede Korkut. The Book of Dede Korkut Qadjar. Iranian destan of Koroğlu. Performance.

\footnotetext{
1 À Florence Goyet.

${ }^{2}$ Centre Urbanisation Culture Société, Montréal.
} 
Le patrimoine commun des Turcs Oghuz ${ }^{3}$, Le Livre de Dede Korkut ${ }^{4}$ compte douze récits autonomes, en vers et en prose, de longueur moyenne et le thème de la guerre n'y est pas dominant. Qu'a-t-il donc en commun avec une Chanson de Roland ou une lliade ? Qu'est-ce qui nous amène à le classer dans la même catégorie, celle de l'épique ? Ce ne sont ni le thème ni la forme dans leur sens classique. C'est quelque chose qui les recouvre sans s'y limiter : l'imaginaire épique. Nous avons déjà abordé la question de « l'imaginaire épique » et à partir de l'analyse des occurrences du syntagme dans un large corpus constitué des écrits universitaires, nous avons proposé de le mobiliser comme un concept opératoire, un outil pour étudier l'épique (Akbarpouran, 2019). Nous avons montré que, dans cette perspective, l'imaginaire n'est pas seulement créé par la grande récurrence de certains thèmes. II est issu d'une synergie dynamique entre le textuel et l'extratextuel; entre la thématique, la médiation particulière qui se charge de sa transmission et sa réception par le public; et, finalement entre les interactions qui lient l'imaginaire social et l'imaginaire épique.

Le Livre de Dede Korkut en tant que destan est une épopée ${ }^{5}$. Et nous savons également que les douze récits développent douze intrigues qui accomplissent ensemble « le travail épique » - notion mise au point par Florence Goyet pour évoquer la fonction sociale de l'épopée (Akbarpouran, 2014). Dans cette étude nous essaierons d'interroger l'imaginaire épique à partir des données concrètes et variées que l'examen du Livre de Dede Korkut nous fournit. D'un côté, 2018 a vu la découverte d'un deuxième ouvrage associé au même cycle épique. Cet ouvrage que nous allons distinguer de l'ouvrage principal - connu sous le titre de Livre de Dede Korkut ou Dede Qorqud - par l'adjectif qadjar ${ }^{6}$ a été écrit deux siècles plus tard que le premier ${ }^{7}$. D'un autre côté, la tradition de la récitation du destan se perpétue jusqu'à nos jours et les aşıq iraniens récitent toujours les destan de Koroğlư ${ }^{8}$, un ouvrage qui a remplacé les destan de Dede. Donc, nous avons l'occasion d'observer et de reconstituer la réception des textes par leur auditoire traditionnel au cours du temps, ce qui

\footnotetext{
${ }^{3}$ La grande confédération tribale qui dans les $X^{e}$ et XIIe siècles a quitté les terres ancestrales, dans l'actuel Kazakhstan, pour migrer vers le sud et l'ouest. Cette confédération se trouve à l'origine de nombreuses dynasties qui ont régné sur le plateau iranien et en Anatolie, comme les Seldjoukides, les Safavides et les Ottomans.

${ }^{4}$ L'origine et la date de formation de ce cycle épique faisant toujours l'objet de controverses, nous y reviendrons.

${ }^{5}$ Sur la discussion critique en Turquie et l'élaboration de la notion d'épopée, voir Akabarpouran, 2017.

${ }^{6}$ Le manuscrit appartenant à un Turkmène de Gonbad, ville située dans le district Turkmen Sahara en Iran, les spécialistes turcoiraniens le qualifient de Dede Korkut de Gonbad ou de Turkmen Sahra. Pourtant, pour un public qui ignore l'intrication des communautés ethnolinguistiques en Iran, cette dénomination peut être à l'origine d'une mauvaise interprétation. D'après les propos de Veli Muhammet Hoca, celui-ci a acheté le manuscrit parmi une série de livres d'un descendant de la dynastie Qadjar à Téhéran. Cette dynastie qui a régné sur l'Iran de 1786 à 1925 était issue d'une tribu appartenant à la confédération tribale oghuz, au même titre que les Seldjoukides et les Ottomans et les populations qui ont fondé le Turkménistan. Il y a assez de différences ling uistiques pour qu'on distingue la branche occidentale des langues oghuz, parlée en Turquie, en République d'Azerbaïdjan et par la majorité des turcophones iraniens, de la branche orientale parlée en Turkménistan et le nord-est de l'Iran (Shahgoli et al. 2019,150). En ce qui concerne la date de la transcription du manuscrit, les notes ajoutées par l'auteur/copiste peuvent nous éclairer : une définition empruntée au dictionnaire Sanglax, rédigé en 1759, nous amène à situer la transcription dans une époque postérieure à cet ouvrage (p. 156-157).

${ }^{7}$ La date et le lieu de la transcription du manuscrit font l'objet de débats. Nous partageons la théorie d'Irène Mélikoff selon laquelle cette version est le résultat des remaniements au XVe siècle d'une œuvre longuement restée orale (Mélikoff, 1995, 18).

${ }^{8} \mathrm{~A}$ ce propos, je me permets de renvoyer à mon livre en cours de publication : Koroğlu du XIXe siècle et les aşıq iraniens.
} 
nous procure un riche éventail d'éléments de comparaison et de réflexion pour creuser la problématique de l'imaginaire épique. Nous aborderons ici trois questions : autour de quels thèmes le récit épique s'articule-til ? En quoi le hors récit et l'extratextuel contribuent-ils à l'actualisation épique d'un récit qui ne contient aucune scène de combat ? Comment dans un contexte traditionnel l'imaginaire, notamment spatial, déployé dans ces destan agissait-il sur la société ?

\section{L'angoisse de la conquête}

Daniel Madelénat distingue deux définitions de l'épopée : « au sens étroit, genre de la tradition occidentale; au sens large, classe de narrations de ton grave, sans spécification de longueur, de mètre, de type d'actions, qui rappelle l'extension de l'épos oral. » (Madelénat, 1986 : 73). Dans cette perspective, « le récit, généralement oral (l'epos), peut charrier des thèmes très différents, et le conte, le chant lyrique, voire le drame, assumer la représentation de la carrière héroïque » (Madelénat, 1986 :14). Les nuances apportées par les études comparatives ont ouvert les portes du sanctuaire du genre épique à de nombreux ouvrages privés du statut littéraire des épopées canoniques occidentales, mais l'analogie entre le récit de la guerre et l'epos reste toujours en vigueur. Certes, la construction de tout récit requiert un élément perturbateur et un dénouement, mais l'epos s'y investit avec un enthousiasme particulier. Il a le potentiel pour convertir toute péripétie apparemment banale en une question de vie et de mort ! Nous reviendrons sur ce sujet plus loin pour savoir comment une récitation réinvestit et mobilise le récit; mais sur le plan narratif aussi la tension est observable.

Articulés autour des scènes de combat ou non, les thèmes qui participent à la mise en intrigue sont tous des thèmes qui interpellent sérieusement et profondément un très grand nombre des agents sociaux, les thèmes associés aux soucis et aux appréhensions collectives. Qu'est-ce qui garantit la perpétuité d'une tradition orale si ce n'est l'intérêt d'un grand public ? Après tout, c'est seulement un public riche et bien placé dans la hiérarchie sociale qui peut organiser une grande cérémonie et commander la récitation par laquelle les récits vivent et se façonnent. En effet, dans le cas des épopées traditionnelles, la mémoire collective endosse le rôle décisif de médiation et c'est elle qui gère le répertoire des thèmes et des intrigues. Pour subsister, ces épopées reposent avant tout sur les cérémonies où les bardes communiqueront leur savoir à l'assistance et où les réussites des bardes encourageront les nouvelles générations à s'initier dans le métier. Sans aucun doute les guerres et les grandes souffrances marquent les esprits et s'incrustent dans la mémoire collective des sociétés : I'historiographie traditionnelle peut l'attester parfaitement. Et cela pas seulement grâce aux mécènes impliqués, mais aussi par l'impact de l'événement sur la vie collective. En effet si Ricœur voit en épopée l'ancêtre commun du récit historique et de la fiction, c'est que l'épopée " préserve la mémoire de la souffrance, à l'échelle des peuples » (Ricœur, 1985, 274-275). D’où vient alors cet engouement des peuples à ruminer les souffrances anciennes ? N'est-ce pas les conflits et les tourments contemporains qui provoquent ce genre de réminiscences, ressuscitent les affres de jadis, actualisant ainsi les 
représentations narratives du passé ? Après tout, pour se faire comprendre, l'épopée "se doit [...] de travailler avec le matériau que tous connaissent » (Goyet, 2006, p. 268-269).

À en croire Paul Ricoeur l'identité, individuelle ou collective, se construit à travers les récits qu'on se raconte à propos de son passé. Mais l'accès au vécu du passé - le nôtre ou celui d'autrui, individuel ou collectif - n'est pas immédiat : sans l'intermédiaire du récit il est impossible.

De même qu'il est possible de composer plusieurs intrigues au sujet des mêmes incidents (lesquels, du même coup, ne méritent plus d'être appelés les mêmes événements), de même il est toujours possible de tramer sur sa propre vie des intrigues différentes, voire opposées. (Ricœur, 1985, 358)

Aussi le récit de vie, tel que conçu par Ricœur, nous rappelle-il la récitation épique perpétuée au sein de la tradition orale : « issue de la rectification sans fin d'un récit antérieur par un récit ultérieur, et de la chaîne de reconfigurations qui en résulte. (Ricœur, 1985, 357-358) Le récit épique peut également accumuler plusieurs événements historiques - décalés les uns par rapport aux autres sur le plan réel - sous forme d’un seul récit. Mais si le récit épique offre à son public le moyen de se faire une identité collective et de la réviser au sein d'une expérience collective et d'un événement social, ce n'est pas seulement pour la transmettre : comme nous l'avons évoqué, la rectification du récit s'effectue en fonction des situations présentes.

Le passé est raconté avec des références aux conditions actuelles puisque le narrateur peut repenser sa biographie en relation à des schémas cognitifs incorporés postérieurement aux évènements racontés, mais antérieurs au moment présent (Lindón, 2005, 59). D'ailleurs toujours d'après Ricœur « le futur est inscrit dans le présent » (Ricœur, 1985, 302). Lecteur de Koselleck, il voit en l'identité le fruit de "l'expérience du passé ", mais également de "l'horizon d'attente », et par ce dernier il entend "l'espoir et la crainte, le souhait et le vouloir, le souci, le calcul rationnel, la curiosité, bref toutes les manifestations privées ou communes visant le futur » (idem). Aussi les transformations qu'on constate au sein d'une tradition orale trouvent-elles d'autres explications que la simple et innocente faille de la mémoire ou l'insouciance de l'assistance à l'égard des dérivations. Reconnaitrons-nous dans les actualisations diverses d'un récit, les projections collectives vers le futur, les matrices des changements au lieu de les interpréter comme les conséquences insignifiantes et indispensables de ces changements ?

Florence Goyet reconnaît dans les « parallèles-différences » déployés dans la Chanson de Roland les argumentations implicites d'une communauté à l'égard des conflits qu'elle vit. Ce qui fait du dénouement une décision sociale, une nouveauté (Goyet, 2006, 211-212). Notre analyse du travail épique dans le Livre de Dede Korkut nous a permis de repérer les parallèles-différences entre les cadets et les ainés (Akbarpouran, 2014) et nous a amenée à la conclusion que chaque jeune garçon qui s'initie à cette aventure et qui gagne le titre du bey, n'est que l'incarnation la plus manifeste d'un mouvement, d'un dépassement de la crise, celle vécue par les Turcomans ${ }^{9}$ installés dans de nouveaux territoires. Le désir de s'adapter à la nouvelle vie et

\footnotetext{
${ }^{9}$ C'est la dénomination déployée pour les populations semi-nomades et issues d'Oghuz pendant plusieurs siècles, après leur conversion à Islam et avant qu'elles n'adoptent chacune de nouvelles dénominations dans de nouveaux territoires.
} 
l'angoisse de perdre tous ses repères se conjuguent pour donner vie à un conflit que l'épopée cherche à résoudre en examinant diverses options, par le moyen de diverses configurations narratives qu'elle développe en parallèle. Les douze récits posent la même question de façons différentes et permettent ainsi au public d'envisager ces enjeux avec des perspectives diverses.

Quant au Dede Korkut qadjar, le manuscrit contient deux récits dont seulement un correspond à la forme habituelle du destan ${ }^{10}$, puisque l'autre est à la première personne ${ }^{11}$. Écrits avec un très grand décalage historique, ils mettent en scène un héros qu'on rencontre déjà dans l'ouvrage précédent : Salur Qazan. Les motifs narratifs montrent eux aussi une très grande affinité avec les motifs de l'ancien ouvrage : 1 . Le protagoniste part à la chasse et une fois éloigné de ses hommes, il se retrouve en face d'un élément menaçant ; 2 . Il abat le dragon ; 3. Avant qu'il n'enlève la peau de dragon qu'il porte, les gens de l'Oghuz le prennent pour un dragon, mais ils finissent par le reconnaître et les retrouvailles se fêtent. Pourtant, ce récit marque un changement de paradigme. Effrayé par le dragon, Salur s'adresse à son tuteur et celui-ci l'éclaire : ce qu'on appelle dragon n'est qu'un grand serpent ! Faut-il voir dans cette scène une rupture heureuse avec le monde chimérique d'un autre temps ? Après tout c'est grâce à ce désenchantement qu'il sort vainqueur. Le semi-récit à la première personne reprend aussi de nombreux motifs anciens pour raconter une nouvelle conquête : celle du territoire que nous connaissons aujourd'hui sous le nom d'Azerbaïdjan iranien, la grande province où régnait le dauphin des rois qadjar.

Les quatorze récits de notre corpus narratif mettent en scène divers éléments hostiles ou conflictuels qui incarnent tous une menace : ennemi, rebelle, traitre, dragon, monstre, la mort. Si le symbole du mal change d'un récit à l'autre, son mode de fonctionnement reste invariable : soit on l'écarte tout de suite et avec toute sa vigueur, soit on perd son honneur et cela débouche sur l'effondrement de toute la confédération tribale. Ainsi mène-on la guerre pour supprimer une menace, mais aussi pour se réaliser et se faire une place dans la société - projet dont la défaite peut avoir des conséquences graves sur la vie collective. Dans le cas de la tradition des destan en Iran, ce qui distingue les destan du Livre de Dede Korkut et les destan de Koroğlu d'autres destan n'est pas seulement les diverses variantes du thème du conflit ou de la crise, mais aussi cette visée collective et une tonalité particulière qui engagent le public dans un conflit grave et qui lui font partager, un peu plus loin, le soulagement et la fierté de le résoudre.

La crise et le conflit représentent un temps « où les anciennes vérités ne sont plus en prise sur le réel, ne suffisent plus à se conduire dans un monde bouleversé " (Goyet \&Vinclair, 2018, 20). Et ce bouleversement $n$ 'est pas seulement vécu dans le passé dont on se raconte le récit, il marque le moment même où l'on fouille dans l'expérience collective et dans les matériaux traditionnels pour choisir le récit à commanditer ou à réciter! Moment où l'on fait le choix du récit et de la version - choix exercé, entre autres, à travers celui du barde - à commanditer; où le barde apporte des nuances dans le récit commandité pour

\footnotetext{
${ }^{10}$ Lignes 730-783.

11 Lignes 706-729.
} 
s'assurer de la réussite de sa performance; et où le public réagit à cette nouveauté : approbation ou rejet. La première s'exprime par les acclamations et les gratifications sur place et l'invitation ultérieure du barde à d'autres cérémonies, le deuxième par les contestations parfois violentes, les questions ou, dans les cas moins graves, par le décrochement lors de récitation et la perte du statut et du marché plus tard.

Nous ne pouvons pas développer une théorie générale du thème épique dans le cadre de cette analyse. Il nous semble néanmoins important de constater qu'au moins dans ces treize récits le thème épique est loin d'être un élément purement textuel qui s'ouvrirait d'une même façon à tout public et à tout moment. Cette observation résonne avec l'approche adoptée par le thématicien contemporain Michel Collot selon lequel «le thème a une structure (textuelle) et un horizon (extratextuel) » (Collot, 1988, 86). De fait, un élément de réponse à la problématique du thème épique peut être cherché du côté de l'horizon extratextuel des thèmes : la tonalité affective des thèmes dans Le Livre de Dede Korkut nous est inaccessible. Les scènes de combat sont les seules instances où nous pouvons cerner l'ampleur et l'intensité investies dans le récit. Pourtant, les gens qui entendaient un aşıq chanter ou réciter dans une cérémonie et partageaient cette identité collective vivaient certainement une autre expérience, comme c'est toujours le cas pour la réception de Koroğlu par les gens auxquels elle s'adresse vraiment, selon une médiation singulière à travers laquelle le récit épique était reçu comme tel. Ainsi, le récit n'aurait-il pas besoin d'une médiation particulière à travers laquelle il sera reçu et plus précisément vécu comme épopée ? Le destan turc s'ouvre à son auditoire avant même que la récitation ne commence. II mobilise tout une cérémonie et s'approprie le space de vie de son public pendant plusieurs soirées. Sous le charme de cette cérémonie le salon, les couloirs et le jardin d'une maison change en une arène où les participants se disputent leur place dans la hiérarchie sociale. Plus on contribue à la cérémonie, plus on se fait respecter par la communauté.

\section{Récit et hors-récit : les enjeux de la cérémonie}

Le spécialiste de la tradition épique turque, Karl Reichl, distingue deux groupes principaux dans les œuvres narratives formées dans cette tradition : épopée héroïque véritable [" the heroic epic proper »] et l'épopée lyrique d'amour [" the lyrical love-epic or romance »]. Dans le même ouvrage et après de longues discussions il admet cependant que le destan se définit surtout en référence au contexte de communication : c'est " un récit qui est exécuté dans un cadre cérémonial [..], dans un style particulier de chant et de récitation et, en règle générale, accompagné d'un instrument" (Reichl, 1992, 124). Effectivement, dans le jargon des aşıq iraniens, il n'y a pas de vocable précis pour distinguer ces deux groupes et le recours au terme arabe ${ }^{12}$ équivalent de l'épique n'est que très rare et très récent. Pourtant, curieusement, ni pour ces professionnels ni pour leur public une récitation de Koroğlu n'a la même valeur que la récitation d'un destan d'amour classique. A priori, elle est beaucoup plus sérieuse et mérite une attention particulière. En effet, pour eux, les récits de Koroğlu ne se distinguent pas des récits d'amour seulement par le fait qu'ils mettent en scène 
les exploits d'un guerrier, mais aussi parce qu'ils sont véridiques et parce qu'ils sont porteurs de valeurs fondamentales. Pour dire autrement, ils les prennent au sérieux beaucoup plus que les autres.

Cette vision des choses peut nous paraitre bizarre : a priori l'histoire de deux amoureux avec de brèves interventions du merveilleux correspondrait à la vérité plus commodément que le récit d'un rebelle possédant un cheval et une épée magique et menant une vie qui dépasse les mesures humaines à presque tout instant ! Plus curieux encore : les scènes de violence et de mœurs d'autres temps ont depuis un moment commencé à gêner le public et les aşıq se donnent beaucoup de peine pour les justifier, ou les suppriment. Comment peuvent-ils concilier cette intervention sur le corpus traditionnel avec leur foi en la véracité des récits et au caractère intouchable de la tradition ? La réponse est très simple : ils le font au nom même de la vérité. Ils nuancent les corpus traditionnels, non pas parce qu'ils pensent qu'il s'agit d'un récit fictif et qu'un récit fictif se modifie facilement; mais bien au contraire parce qu'ils essaient d'ajuster le récit à un régime de vérité qui le transcende. Cette vérité est d'un autre ordre que celle d'un récit historiographique : loin d'être vérifiable et réfutable, le récit traditionnel a abondamment recours à la fiction, mais ne perd en rien son statut canonique et autoritaire. Pour son public, il n'appartient pas au domaine de la littérature, ni à I'histoire ! Nos observations prouvent bien qu'en Iran contemporain les gens - ceux qui l'apprécient comme ceux qui le trouvent obsolète - ont du mal à accepter l'appartenance d'un destan à l'une de ces catégories. Ici le rapport de l'épique et de la vérité se calque sur celui qui existe entre celle-ci et la tradition même (Boyer, 1986). D’où cette illusion d'exhaustivité et d'atemporalité, et cette adhésion semi-religieuse du public traditionnel qui s'y reconnaît.

L'examen des récitations de Koroğlu dans les milieux traditionnels et les villages iraniens nous a permis de remarquer qu'écouter une récitation traditionnelle est plus que lire un récit à haute voix : 1 . un récit raconté ou récité par une autre personne qu'un barde ne relève pas du même genre ; 2 . pour devenir barde, il faut remplir un grand nombre de critères et accomplir un parcours assez exigeant. Ce qui garantit non seulement le parfait apprentissage du corpus, mais aussi et surtout l'adhésion infaillible de cette personne aux valeurs et à l'imaginaire en question, de façon à ce qu'il devienne l'incarnation même de la tradition et gagne une autorité sociale très considérable! 3 . la récitation du barde s'intègre dans une cérémonie codifiée, et raconter le récit ne constitue qu'une partie des tâches de ce porte-parole des ancêtres ; 4. le barde ne se contente jamais de déclamer le récit brut, à chaque récitation. Il le recontextualise en ayant recours à des explications, des anecdotes, des proverbes, etc. Chaque récitation se construit avec de nombreux sous-genres, mobilisant ainsi une grande partie de la tradition.

Autrement dit, non seulement il y a de nombreux éléments extratextuels qui accompagnent chaque récit et influencent sa réception, mais il y a aussi un grand nombre d'éléments textuels, hors récit, qui sont indispensables à la récitation. Pour nous faire une idée de la réception du Livre de Dede Korkut par son public, référons-nous aux récitations traditionnelles contemporaines de Koroğlu qui ont lieu dans les fêtes de mariage. Bien que depuis très longtemps un mariage requiert le recours aux administrations ainsi qu'aux 
mollâs, un aşıq traditionnel se charge toujours d'ajouter des petites touches de bon augure : donner la bénédiction aux mariés, faire le vœu de mariage aux célibataires dans le public ou dans la communauté, prier Dieu pour qu'il bénisse les mariés et tous ceux qui sollicitent l'aşıq en leur octroyant de bons descendants. II est également sollicité pour intervenir dans les conflits qui peuvent entraver le mariage et la réussite de la cérémonie : il se porte alors juge dans un conflit et réconcilie deux adversaires associés aux mariés, ou se porte garant pour arracher l'accord du père de la fille ou les parents de deux amoureux, etc. Ce qui accorde au barde cette autorité, c'est le statut canonique de la tradition qu'il incarne. D'autant plus que lors de ces interventions, il cite souvent des formules et des motifs empruntés aux récitations.

Ajoutons à ces matériaux extratextuels ceux qui font plus étroitement partie de la récitation, mais sortent du cadre du récit et font appel aux autres genres ou sous-genres folkloriques : I'explicitation de la signification allégorique d'un passage ou de la leçon morale qui appuie la sagesse délivrée par celui-ci ; la mention d'un exemple contemporain pour un geste ou un événement ; la paraphrase et l'explication des expressions et des mots tombés en désuétude ; l'introduction d'un dicton et l'anecdote qui exprime son origine; la localisation d'un toponyme fictionnel en faisant appel aux expériences personnelles et/ou aux éléments connus de son public. D'un point de vue littéraire on serait tenté de caractériser ce corpus de paratexte, pourtant ce mot ne représenterait jamais l'intérêt qu'un auditoire traditionnel y porte. En effet, la plupart des gens qui commanditent un récit le savent par cœur et s'ils payent, c'est surtout pour participer à son actualisation. Le hors-récit suggère un échantillon de la sagesse ancestrale et le récit offre l'occasion de travailler sur et à partir de ces matériaux et de sonder les possibilités qui s'offrent. Du reste, ces éléments textuels recadrent l'histoire racontée et lui ajoutent une touche personnelle, ce qui ouvre la voie à l'alternance des verbes conjugués au présent et au passé. Ces allers-retours entre l'instant de la récitation et un moment indéterminé et mythifié dans I'histoire se conjuguent à la magie d'une réception collective ritualisée et à la capacité d'un barde à créer une synergie singulière et une adhérence maximale.

La mise en espace de la performance contribue aussi largement à renforcer le statut charismatique du barde jusqu'à transformer la cérémonie en une compétition très discrète pour entrer en contact avec celui-ci et prendre part à la récitation. Le barde est la seule personne qui a le droit d'être debout pendant la cérémonie. Même le musicien qui l'accompagne quand il chante est assis par terre. Le barde marche et parcourt le salon. Pendant la cérémonie il ne sort du salon que très rarement : pour un bref repos ou bien à la suite d'un appel pour recevoir une commande de la part de quelqu'un qui est installé à l'extérieur. Les interlocuteurs principaux, eux, sont toujours logés dans le salon. Autrement dit, les couloirs et le jardin de la maison sont réservés aux gens qui ne sont pas en mesure de passer une commande : les femmes, les enfants et les membres marginalisés de la communauté. Dans le salon par ailleurs, les places n'ont pas la même valeur : les invités les plus respectueux sont logés le plus loin possible de la porte et ils sont tout de suite repérables. 
Dans une telle perspective, toute échange avec le barde jouit d'un intérêt symbolique très fort : pour le faire approcher et s'entretenir avec lui, il faut passer une commande. Ce qui exige un minimum de capacité financière et une connaissance adéquate du corpus. N'oublions pas que si quelqu'un commande un morceau qui déplait au public, on lui reprochera son mauvais goût ; et si quelqu'un installé dans le couloir sait passer plusieurs commandes intéressantes, il y a de grandes chances qu'il soit conduit dans le salon la prochaine fois. C'est dans cette ambiance organisée autour du savoir et du pouvoir que le destan turc prend corps et agit sur tout un réseau de relations sociales.

Il ne serait pas abusif d'imaginer que la récitation de Dede Korkut avait aussi recours à ce genre de sagesse hors récit et bénéficiait ainsi d'une réception plus valorisante et plus efficace. En effet, les manuscrits qui nous sont parvenus contiennent en grand nombre des formules et des passages très similaires à ceux qui sont exploités par les aşıq contemporains comme des composantes hors récit de la récitation. La version ancienne, présentée sous forme du "livre " sépare les douze récits des quelques pages consacrées à ce corpus et placées au début du recueil comme un prologue, tandis que dans le Dede Korkut qadjar le récit constitue seulement un tiers du texte. Cette modeste partie succède à une série de passages en vers ou en prose hautement rythmée, ayant un caractère moralisant et/ou relevant de l'éloge. Évidemment dans aucune de deux versions il n'existe de dénomination qui indique qu'il s'agirait d'un prologue ou d'un corpus moins important que le récit. Ici une comparaison entre les coutumes de la mise en écriture plus récente peut être révélatrice. Aujourd'hui et depuis les plus anciens manuscrits légués par les aşıq à leurs disciples, ce sont seulement les parties en vers du récit qui sont consignées. Le reste, s'apprend sur place et pendant les cérémonies.

Autrement dit, dans de tels contextes, l'écriture n'est qu'un support de mémoire pour conserver les parties dont la forme doit rester absolument intouchable. Ce qu'un barde traditionnel n'explique jamais par la position essentielle de telles parties au détriment du reste. Bien au contraire, les maitres conviennent d'exprimer leur désapprobation à l'égard de ceux qui se contentent de chanter ou de réciter ces parties sans parler de la sagesse et sans incarner cette sagesse dans leurs gestes quotidiens, les gens qui se laissent réduire au statut d'un « chanteur »!

Cette conception de la profession converge avec les observations faites par les anthropologues sur le terrain en Afrique où les mots du barde sont censés expliquer le monde et agir sur les individus, où l'épopée épouse la valeur d'une "encyclopédie tribale » (Diouf, 1991, 31) et va encore plus loin pour remplir la fonction d'une "institution sociale» (Seydou, 1996). D'ailleurs, depuis les années 1990 les études anthropologiques et littéraires attestent la parenté qui existe entre la tradition de récitation des bardes en Asie centrale et le chamanisme préislamique (Basilov, 1992 \& Reichl, 2001\& Reichl, 2003). Le même destin, l'islamisation du chamanisme, s'observe dans la branche occidentale de la tradition en Anatolie (Zarcone, 2013). Quant aux aşıq iraniens, l'affiliation d'une grande partie de leur corpus aux ordres ésotériques est attestée. Et nos observations complètent ce tableau où nous constatons une cérémonie plutôt qu'un récit : 
un genre qui ne se réalise qu'à travers la performance présidée par une figure d'autorité et l'incarnation de la tradition, un barde.

Une telle cérémonie n'a pas seulement vocation à actualiser et à transmettre la tradition, mais se doit aussi de rassembler les membres de la communauté et de les aider à se situer par rapport aux autres. C'est grâce à cet " acte performatif » et à la " cérémonie de reconnaissance ", pour reprendre l'expression de Pierre Vinclair, que les membres de la communauté se reconnaissent comme tels et tissent des liens de complicité (Vinclair, 2014, 391-410). La reconnaissance peut être le mot-clé d’une récitation d'aşıq, car il s'agit également de la reconnaissance des valeurs, des morales enseignées par le barde, de l'histoire collective qu'il re-raconte, de l'identité collective dont il trace les frontières, voire des thèmes qu'il met en avant comme les sources de soucis et d'espoir. La cérémonie et le corpus malléable et foisonnant qu'elle met en chantier investit et réinvestit le récit en le plongeant dans la source de la tradition. C'est à ce moment où il « se laisse déborder » (Goyet \&Vinclair, 2018, 20) qu'il travaille la société.

\section{L'intégration de l'innovation : nouveaux territoires, nouvelles altérités}

Nous avons vu que l'imaginaire épique qui soutient le Livre de Dede Korkut déploie certains thèmes grâce à un récit et dans un cadre cérémonial et ritualisé. Le statut canonique de la tradition où s'ancre le récit et la récitation leur accordent une valeur normative au plan social. Néanmoins, la dimension prospective de l'identité narrative n'arrête pas d'actualiser le récit et par-delà, c'est la tradition même qui s'actualise. En tant qu'une des médiations culturelles les plus accessibles à tous les membres de la société et loin de se réduire à un simple divertissement, cette épopée sait bien se servir de l'autorité du passé et de la tradition, aussi bien que de l'intérêt socio-politique des problèmes graves résolus dans le passé au travers des guerres, des sacrifices et des massacres. Raconter les épreuves que les beys oghuz dépassaient dans un « autrefois » mythifié pour arriver au pouvoir et le garder fait circuler une connotation de crise et interpelle, réactivant ainsi d'innombrables éléments socioculturels et politico-religieux associés à ce sentiment dans la mémoire collective.

L'épopée s'impose, dans cette perspective, comme une partie de la tradition et contribue alors à la reconstruction permanente de l'imaginaire social. Ricœur affirme : « Tout se passe comme si cet imaginaire [l'imaginaire social] reposait sur la tension entre une fonction d'intégration et une fonction de subversion » (Ricœur,1984, 53). La fonction intégratrice et unificatrice de l'épopée a toujours été évidente, mais pour cerner sa fonction novatrice il faut se rendre compte du travail de construction et de reconstruction permanente qui se profile derrière les entités que l'on considérait invariables et uniques : histoire, tradition et identité.

Pascal Boyer qui qualifie la tradition de discours narratif (Boyer, 1984) avance l'idée que l'épopée, tout en s'enracinant dans ce discours, le contredit en mobilisant une partie du savoir commun, et lui apporte des nouveautés au bout d'un certain temps (Boyer, 1982). La réalité reconstruite qui se renouvelle à chaque 
réception relève de la mémoire collective comme le résultat de la gestion du passé selon " un mécanisme qui choisit, garde, rejette et transforme les principaux éléments d'un passé culturel » (Van Gorp \& MusarraSchrøder, 2000, 27). La tradition et le récit épique ne font que mobiliser une partie du savoir commun pour qu'elle concorde avec la nouvelle vérité sociale qui se fait jour et le thème y joue un rôle déterminant : il « rend pertinente une partie de notre stock de connaissances et focalise notre attention sur elle » (Moscovici \& Vignaux, 1994, 37). Rien d'étonnant alors que faire face aux conflits encourage une représentation du passé marquée par un élément hostile. Les thèmes, les thêmata pour reprendre le vocabulaire de Moscovici, font des liens entre les individus et l'expérience collective, et par-delà entre les générations, les membres d'un groupe ou les groupes d'une société. Ils « se réfèrent à des possibilités d'action et d'expérience en commun et intégrés à des actions et des expériences passées. [...] ils sont toujours préservés comme des sources constantes de nouvelles significations ou de combinaisons de significations si besoin est » (idem).

Le Livre de Dede Korkut se réclame de l'oghuznâme ${ }^{13}$, la tradition qui célèbre la vie d'Oghuz khan, l'ancêtre mythique des Turcs oghuz et celle des héros de cette confédération. Il reprend de nombreux motifs, formules, personnages et valeurs repérables dans les oghuznâme qui nous sont parvenus dans la tradition écrite. Les deux moments différents de l'histoire de la tradition que nos deux ouvrages ont captés montrent de grandes divergences ainsi qu'un rapport différent à la tradition écrite. Nous pouvons voir dans ces ruptures les suites des événements historiques, mais également et surtout les nouveautés apportées par I'imagination narrative. Ce qui est important, c'est de faire état du caractère subtil des changements : ce sont les nuances subtiles qui donnent au bout d'un temps ces changements, voire ces ruptures. L'observation des transformations dans les destan de Koroğlu ne laisse aucun doute en ce qui concerne l'existence d'une telle procédure : aucune nouveauté n'intègre la tradition, sauf si elle passe inaperçue ou si elle se justifie habilement au nom du retour à une étape antérieure et plus correcte de celle-ci.

La rupture la plus brutale qui s'observe entre les deux ouvrages concerne l'altérité et le territoire de I'Oghuz. La métamorphose de la tradition favorise, aux XV-XVIe siècles et dans les territoires directement ou indirectement concernés par la conquête de l'Anatolie, la formation d'un recueil où l'identité de l'Oghuz, nomade, se définit par opposition aux chrétiens propriétaires des forteresses et évoqués comme mécréants, tandis que le même matériel traditionnel s'incarne deux siècles plus tard sur le plateau iranien de façon à opposer son public aux Persans ${ }^{14}$.

Le passage qui assoit cette altérité est balisé par une formule particulière qui lui vaut le statut d'une série de prédictions effectuées par Dede Korkut en personne : « Moi qui suis Dede Korkut, je n’ai pas vécu ce temps, mais je vous en parle comme si je l'avais vécu. ». Une prédiction qui s'impose comme avertissement contre les mixités culturelles avec les autochtones. Bien que dans le Livre de Dede Korkut épouser les princesses mécréantes soit une pratique souhaitée et conseillée, dans le Dede Korkut qadjar l'épouse

\footnotetext{
13 Pour plus d'information sur ce genre et ses transformations voir Kincses-Nagy, 2019.

${ }^{14}$ Voir lignes 581-632. Le mot utilisé dans le livre est tat, le terme par lequel les Turcs désignaient et désignent encore dans certaines régions les populations parlant divers dialectes des langues iraniennes dont le plus connu est le persan.
} 
persane est l'objet de méfiance : elle ne respecterait pas les coutumes comme il faut et de telles attitudes accableraient, par là même, l'homme et le clan qui l'accueilleraient. Pire encore, les descendants de tels mariages auraient des problèmes congénitaux et mèneraient une vie monstrueuse ${ }^{15}$. Cet avertissement fait écho à un thème qui structure le Livre de Dede Korkut et revient également dans les récitations de Korkğlu : la succession des héros par les descendants dignes de ce nom. Ce souci dépasse largement celui de la guerre contre l'ennemi ou un monstre. Si tous les récits du Livre de Dede Korkut se ferment sur la célébration du triomphe, la crise et le conflit ne manquent pas d'apparaître dans un autre récit et sous une autre forme. Se créent alors plusieurs récits en parallèle où les héros suppriment les ennemis, punissent les rebelles et les traîtres ou abattent un monstre.

L'adversité et l'hostilité que manifestent un dragon, un monstre ou un mécréant sont immédiates et évidentes. Il s'agit d'un objet de haine à abattre et les héros y parviennent plutôt facilement. Cependant, cet élément hostile revient d'un moment à l'autre et ne se laisse pas éliminer. Les héros ont sans cesse besoin de se faire reconnaitre par leur communauté comme un membre fidèle et valide; de même qu'ils se préoccupent de la succession de leur héritage de guerrier noble. Le Livre de Dede Korkut témoigne de I'installation des tribus turcomanes en Anatolie et au Caucase, une aventure imprégnée de la fierté d'être les héros du monde musulman et de l'angoisse de l'acculturation. La même préoccupation s'observe dans le Dede Korkut qadjar où il faut faire des territoires évoqués, les villes et villages iraniens, un territoire oghuz sans avoir à s'assimiler aux populations autochtones iraniennes. Il est difficile de dire si ce vœu a été exaucé ou non. L'Empire qadjar a duré plus de deux siècles et cela avec beaucoup de succès ; la turcisation du pays aussi a continué pendant tout ce temps. Pourtant, tout a évolué : les mœurs, les valeurs et même l'identité d'Oghuz. Il arriva un moment où le destan même qui pouvait tenir en haleine les milieux populaires turcs n'était plus celui de la noblesse oghuz, mais celui d'un rebelle contre celle-ci. Au XIXe siècle qui a vu consigner le plus ancien manuscrit de Koroğlu à partir d'une récitation à Tabriz (Chodzko, 1842), le cycle de Dede Korkut a été oublié par les aşıq. Après tant de déception subie par les Turcomans et la fusion massive des cultures dans des Empires cosmopolites fondées par les noblesses oghuz, la fidélité à son égard et à une grande partie des valeurs tribales ne semblait plus avoir aucun sens ${ }^{16}$ ! Le recours du copiste du manuscrit de Dede Korkut qadjar à un dictionnaire pour commenter un passage nous informe sur la réception de cette épopée dans cette époque. Le copiste n'était pas assez familier avec les tournures et il ne transcrivait pas la performance d'un barde qui pourrait le renseigner sur son corpus. Ce n'aurait pas été le cas si le corpus était toujours récité par les bardes et connu d'un grand public. Il s'agit probablement de la réécriture d'un ouvrage déjà devenu ancien, d'une entreprise d'élite liée à la cour pour préserver une œuvre d'intérêt généalogique.

\footnotetext{
15 Voir lignes 605-623.

${ }^{16}$ Sur le destin de ces Turcomans en Iran et les éléments qui contribuent au succès de Koroğlu, je me permets de renvoyer à un article en cours de publication : « Le destan de Köroğlu et l'éloge de la rébellion ».
} 


\section{Conclusion}

L'imaginaire épique s'épuise dans la tradition et l'actualise. C'est une façon de faire face aux crises et aux changements déstabilisants dans un temps et un milieu où n'existaient pas les institutions modernes qui se partagent les tâches de réfléchir sur le monde et de produire des savoirs que les gens doivent incorporer pour faire partie d'une société. La tradition était la source de la sagesse et entretenait des liens étroits avec l'au-delà et le passé. Les exploits des héros chers à cette société figuraient, certes, en tête de liste des intrigues dont le récit endossait la vocation de préserver et de transmettre cette sagesse. Mais dans les deux ouvrages appartenant au cycle de Dede Korkut, derrière les scènes de réussite flagrante contre l'ennemi et les monstres se profilent des inquiétudes profondes, qui ont plutôt trait à la vie civile. C'est grâce à la configuration narrative et à d'innombrables façons d'envisager la question que la communauté s'interroge sur les crises qu'elle vit. Cependant, le récit ne pouvait pas agir tout seul et il fallait toute une institution pour accomplir une mission de si grande ampleur.

Comme l'observation des récitations d'aşıq le confirme, les bardes et les cérémonies qu'ils présidaient constituaient cette institution : ainsi le récit était développé dans un cadre composé par de nombreux éléments que l'on peut répartir en deux groupes : le hors-récit et l'extratextuel, ce qui favorisait une révision de la tradition pour mobiliser certains thèmes correspondant aux affects qui habitaient la communauté et pour interroger le public. Relevant de la tradition, l'imaginaire épique réinvestissait celle-ci par de nouveaux éléments pour ouvrir la voie à de nouveaux horizons.

\section{Références bibliographiques}

AKBARPOURAN, M. Koroğlu du XIXe siècle et les aşıq iraniens, Istanbul :Isis, 2021.

AKBARPOURAN, M. (En cours de publication). Le destan de Köroğlu et l'éloge de la rébellion. In: Actes du colloque de Rouen du REARE, Rouen, 2018.

AKBARPOURAN, M. Vers l'étude du «travail épique» dans le Livre de Dede Korkut. In: Études mongoles et sibériennes, centrasiatiques et tibétaines, Université Paris-X Nanterre., Paris, 45, 2014.

AKBARPOURAN, M. Le destan turc est-il une épopée ? Premiers débats et prolongements actuels. In: Le Recueil Ouvert| Projet Épopée, Université Grenoble-Alpes, 2017.

AKBARPOURAN, M. Approches de l'imaginaire épique; vers la conceptualisation d'une notion. In: Bulletin de l'IFAN Ch. A. Diop, sér. B, 59(1-2), 2019, p. 157-167.

BAZIN, L., \& GOKALP, A. Le livre de Dede Korkut. Récit de la Geste oghuz, Gallimard, Paris, 1998.

BOYER, P. Récit épique et tradition. In: L’homme, I'EHESS, Paris, 22 (2), 1982, p. 5-34.

BOYER, P. La tradition comme genre énonciatif. In: Poétique (Collection), Seuil, Paris, 58, 1984, p.233-251.

BOYER, P. Tradition et vérité. In: L’homme, I'EHESS, Paris, 26 (97/98), 1986, p.309-329.

CHODZKO, A. Specimens of the Popular Poetry of Persia (as found in the Adventures and Improvisations of Kurroglou, the bandit-minstrel of Northern Persia). Harrison And Sons, London: 1842.

COLLOT, M. Le thème selon la critique thématique. In: Communications, Seuil, Paris, 47 (1), 1988, p.79-91. 
DIOUF, M. L'invention de la littérature orale : les épopées de l'espace soudano-sahélien. In: Études littéraires, 24 (2),1991, p.29-39.

GOYET, F. Penser sans concepts : fonction de l'épopée guerrière. Iliade, Chanson dee Roland, Hôgen et Heiji Monogatari. Honoré Champion: Paris, 2006.

LINDON, A. Récit autobiographique, reconstruction de l'expérience et fabulation : une approximation à I'action sociale. In: Sociétés, Québec, 1, 2005, p. 55-63.

MADELENAT, D. L'épopée, PUF : Paris, 1986.

MOSCOVICl, S., \& Vignaux, G. Le concept de thêmata. Structures et transformations des représentations sociales. In : Structures et transformations des représentations sociales, Christian Guimelli (eds), Delachaux et Niestlé, Loney (Suisse), 1994, p. 25-72.

REICHL, K. Turkic Oral Epic Poetry: Traditions, Forms, Poetic Structure. Londres: Routledge, 1992.

REICHL, K. L'épopée orale turque d'Asie centrale : Inspiration religieuse et interprétation séculière. In: Études mongoles... et sibériennes, Université Paris-X Nanterre., Paris, 32, 2001, p. 7-162.

REICHL, K. The search for origins: Ritual aspects of the performance of epic. In: Journal of Journal of historical pragmatics, John Benjamins Publishing Company, Pays-Bas, 4 (2),2003, p. 249-267.

RICCEUR, P. L'idéologie et l'utopie : deux expressions de l'imaginaire social. In: Autres Temps : les cahiers du christianisme social, Paris, 2 (1), 1984, p.53-64.

RICCEUR, P. Temps et récit III. Paris : Le Seuil, 1985.

SEYDOU, C. L'épopée, genre littéraire ou institution sociale ? L'exemple africain : L'épopée : mythe, histoire, société. In : Littérales, Université Paris Nanterre, Paris, (19), 1966, p.51-66.

SIAFLEKIS, Z.I. La mémoire déformatrice imitation et transformation dans la parodie de l'épopée homérique: XVII ${ }^{e}-X V I I I{ }^{e}$ siècle. In: Genres as repositories of cultural memory, Van Gorp, H., \& Musarra-Schrøder, U. (eds.), Rodopi, Amsterdam, 15 (5), 2000, p. 27-36.

VINCLAIR, P. De l'épopée et du roman : énergétique comparée. Thèse doctorale, Littératures. Université du Maine, 2014.

WILKS, J. M. Issues of genre and form in Turkic heroic works. In: Turkish Language, Literature, and History: Travelers' Tales, Sultans, and Scholars Since the Eighth Century, Hickman, B. \& Leiser, G. (eds.), Londres: Routledge, 2015, p. 343- 357.

ZARCONE, T. Shamanism in Turkey: Bards, Masters of the Jinns, and Healers. In: Shamanism and Islam: Sufism, Healing Rituals and Spirits in the Muslim World, Zarcone, T., \& Hobart, A. (eds), London: I.B. Tauris, 2013, p. 169-202.

MELIKOFF, I. De l'épopée au mythe. Itinéraire turcologique. Istanbul : Isis, 1995.

KINCSES-NAGY, É. The Islamization of the Legend of the Turks: The Case of Oghuznāma. in: Studia UraloAltaica : Competing narratives between nomadic people and their sedentary neighbours :, 53, 125-136. 2019.

SHAHGOLI, N. K., YAGHOOBI, V., AGHATABAI, S., \& BEHZAD, S. Dede Korkut Kitabı'nın Günbet, YAZMASI: iNCELEME, Metin, Dizin ve Tıpkıbasım. Modern Türklük Araştırmaları Dergisi, 16 (2), p. 147-379. 


\section{Annexe 1 : présentation des récits}

\section{Le récit de Boğac Khan le fils de Dirse Khan}

Ce récit met en scène la naissance éperdument attendue d'un jeune garçon et son premier combat. D'après les usages de l'époque, les garçons n'avaient pas de prénom avant de réaliser un haut fait. Alors c'est après ce combat que Dede Korkut donne son prénom à Boğac. Cette reconnaissance sociale annonce sa future succession à son père, seigneur de grande autorité. Déstabilisés par ce changement des rapports de forces, les quarante compagnons du père fomentent un complot. Ils accusent le jeune garçon de la rébellion et encouragent le père à le tuer.

Plus tard, ils ligotent leur seigneur pour le livrer aux ennemis, les mécréants sanglants. Ayant miraculeusement survécu, le garçon de Dirse Khan se précipite à la recherche de son père. Après une longue scène de retrouvailles, il punit les traitres et les ennemis. Nous pouvons repérer dans ce récit quelques éléments dont les variations reviennent dans les autres récits : la chasse, l’ennemi mécréant, la trahison, le rapport ambivalent que le père entretient avec les manifestations du pouvoir chez son fils, la lâcheté qui s'enracine dans la jalousie et le rôle crucial de la mère dans la gestion des conflits entre le fils et le père, les retrouvailles, la reconnaissance.

\section{Le récit où l'on pille la maison de Salur Qazan}

Le deuxième récit s'ouvre sur une scène de fête à la suite de laquelle Salur Qazan, enivré, décide d'aller à la chasse et charge son fils de veiller sa tente. Informés par leur espion, "les mécréants » font une razzia et pillent la tente. La femme, la mère et le fils de Qazan se font capturer et tous les troupeaux de chevaux et de moutons sont confisqués. À la suite de nombreux événements qui visent à humilier Qazan, il finit par vaincre son ennemi et récupérer sa famille. Le mot-clé dans ce récit est l'honneur.

\section{Le récit de Bamsi Beyrek le fils de Qam Büre}

Le troisième récit compte trois scènes de combat dont la première représente la lutte de Beyrek et de la fille avec laquelle ses parents l'ont fiancé, enfant. Ils sont tous les deux nés à la suite des vœux que leurs pères ont échangés : marier leurs enfants l'un à l'autre. L'affrontement des deux jeunes les amène à se reconnaitre et à tomber amoureux. La deuxième scène de combat s'organise quand les mécréants attrapent Beyrek dans la tente nuptiale pour l'éloigner de la jeune femme. Seize ans après la captivité de Beyrek, le seigneur mécréant amoureux de la jeune femme parvient à la persuader de la mort de Beyrek et la fait se préparer au mariage. La troisième raconte le retour de Beyrek qui se libère juste à temps pour tuer son rival et épouser sa fiancée. 


\section{Le récit où Oruz Bey le fils de Qazan Bey se fait capturer}

Comme le titre l'indique, le récit met en avant le thème de la captivité. Embarrassé par le fait que son jeune garçon n'a pas encore versé du sang, Qazan organise la première chasse de son fils. Informés par leur espion, les mécréants les surprennent et capturent le jeune héros. En compagnie de ses quarante compagnons et sa femme, Qazan bat les mécréants et libère son fils.

Un autre élément à retenir dans ce récit, c'est le parallélisme qu'il établit entre verser le sang de l'ennemi et verser du sang lors de la chasse.

\section{Le récit de Deli Dumrul le fils de Doha Qoca}

Le jeune brave qu'est Deli Dumrul apprend pour la première fois de sa vie la nouvelle de la mort d'un brave et lance un défi à l'assassin inconnu. Le thème de la mort adversaire qui a une brève présence au début du récit reste latent jusqu'au bout. Pour affronter cet ennemi, le jeune brave commence par guerroyer. En vain. Dans une deuxième étape, il reconnaît l'existence de Dieu tout-puissant et le supplie. II ne peut s'échapper qu'en trouvant quelqu'un qui mourra à sa place. Ses parents lui refusant ce sacrifice, le brave s'apprête à mourir. Mais sa femme se porte volontaire pour le remplacer, proposition que le jeune homme repousse. Le couple choisit de mourir ensemble et attendri par cette scène, Dieu leur donne une longue vie à tous les deux.

Il est à remarquer que le récit n'a aucun recours aux certains éléments repérables dans la plupart des récits : mécréance, jalousie, traîtrise, guerre et armée. Toutefois un examen plus approfondi peut nous amener à découvrir les analogies: le jeune brave qui lance un défi à la mort correspond bien au type du rebelle. Le refus par les parents de donner leur vie trouve son équivalent dans la traîtrise des membres de la tribu. Et, finalement, la longue vie octroyée par Dieu peut justement faire penser au prénom que gagne Boğac et aux titres de noblesse que les autres héros obtiennent.

\section{Le récit du fils de Qan Turali le fils de Qanli Qoca}

Ayant pour point de départ la décision de Qanli Koca de marier son jeune fils et de le préparer à lui succéder, ce récit relate l'affrontement de Qan Turali et de la famille de la princesse mécréante dont il gagne la main dans la lutte contre trois animaux sauvages et dangereux. C'est à la suite du triomphe du beau brave que la fille tombe amoureuse de lui. Les conditions remplies, les mécréants ne peuvent plus empêcher le jeune héros d'emmener sa fiancée. Pourtant après leur départ, ils les poursuivent et d'autres affrontements s'enchainent. 


\section{Le récit de Yegenek, le fils de Qazliq Qoca}

Enivré dans une fête, Qazliq Qoca entreprend une guerre sainte contre les mécréants. Pourtant, au bout d'un court combat singulier avec le prince, le bey turc se laisse emmener comme captif dans la forteresse. Le petit garçon de Qazliq Qoca atteint ses quinze ans en l'absence du père. C'est à cet âge que le jeune garçon découvre l'existence de son père captif. II conquiert la forteresse et libère son père. L'idée de la première guerre y est latente. Comme cela est le cas dans bien d'autres récits, cette idée s'associe à au thème de la relation conflictuelle qui existe entre un bey et son successeur.

\section{Le récit où Basât abat le Cyclope}

L’origine archaïque de certains éléments et la symbolique caractérisent ce récit. Aruz retrouve son fils Basât, perdu il y a longtemps et élevé par un lion. Grâce aux conseils du Dede Korkut et grâce au nom que celui-ci donne à l'enfant, il accepte de revenir dans la tribu. Parallèlement, un cyclope se fait adopter par Aruz. Il est le fruit du viol d'une fée par le berger d'Aruz et incarne la malédiction que sa mère a lancée sur le peuple oghuz. Il se met à manger les nez ou les oreilles de ses camarades et finit par être chassé de la maison. Ce parallélisme met en avant, dans un premier temps, le thème de l'adoption : le Cyclope qui a une moitié surhumaine n'arrive pas à s'intégrer dans la famille et cause beaucoup d'ennuis, tandis que Basât adopté par un lion retrouve « sa » famille et s'y intègre bien. Le deuxième thème à retenir dans ce récit apparaît dans le passage suivant la chasse du Cyclope où la fée lui apparaît et le rend invulnérable en lui passant une bague magique au doigt. L'invulnérabilité du Cyclope qui est déjà devenu un monstre renforce ce parallélisme : seul Basât aura l'honneur de tuer ce monstre. II recevra ainsi le titre de bey. Le Cyclope qui mange deux hommes par jour s'impose comme un ennemi de la tribu tout entière. Il pourrait, selon le texte, causer la disparition de l'Oghuz. Évoquée dans d'autres récits à travers les thèmes plus proches de la vie réelle - infertilité, descendant indigne et inadéquat ou attaque des mécréants qui pillent et capturent les gens de l'Oghuz -, l'angoisse de la disparition trouve son incarnation parfaite dans ce monstre mangeur d'hommes, un rebelle dont la moitié surhumaine l'emporte sur son appartenance tribale.

\section{Le récit d'Emren, le fils de Begil}

La peur de ne pas avoir un bon allié ou un successeur digne revient dans ce récit, mais se voit tout de suite apaisée. Begil qui s'est cassé la jambe dans la chasse, se plaint de ne pas avoir un frère aîné ou un fils pour l'aider. À ce moment-là son jeune garçon l'entend et le réconforte en l'assurant qu'il peut combattre pour son père. Informés par leurs espions de la blessure de Begil, les mécréants attaquent sa maison, mais Emren les massacre. II est intéressant de constater que Begil se considère comme un homme sans descendant bien qu'il ait un fils. Sans doute parce que celui-ci n'a encore accompli aucun haut fait. Cette guerre contre les mécréants qu'il gagne à l'aide de l'ange Gabriel sera la première fois que le jeune héros verse du sang ! 


\section{Le récit de Segrek, le fils d'Uşun Qoca}

Ce récit s'ouvre sur un épisode qui éclaire tout l'ouvrage : Egrek bouscule les beys pour s'asseoir à une meilleure place et se fait reprocher par l'un d'entre eux : « tous les beys qui ont leur place ici, ils la doivent à leur épée [...] as-tu donc coupé des têtes, versé du sang, nourri l'affamé et habillé ceux qui sont en guenilles ? " (Gökalp \& Bazin, 1998, 205). C'est pour gagner sa place qu'Egrek entreprend une razzia et finit en captivité. Les années passent et un événement semblable amène son frère cadet à découvrir l'histoire d'Egrek : « si tu es si brave, tu n'as qu'à aller [...] délivrer ton frère " (Ibid. 206), lui reproche-t-on. Segrek vainc les mécréants et libère son frère. Dans ce récit aussi, les ennemis cherchent à profiter du fait que les deux parents ne se reconnaissent pas pour les confronter. Mais les deux braves finissent par se retrouver et s'allier contre leur ennemi.

\section{Le récit où Qazan se fait capturer et Oruz le libère !}

Le thème de captivité revient dans ce récit et s'associe comme dans d'autres récits au thème de la chasse ou la razzia. C'est lors de la pratique de la fauconnerie, en poursuivant son faucon que Qazan approche la forteresse et se fait capturer. Plus tard le fils de Qazan prend conscience de l'existence d'un père capturé et vient à sa recherche. Dans ce récit aussi l'affrontement, la reconnaissance, la complicité et le triomphe s'enchaînent.

\section{La rébellion de l'Oghuz intérieur contre l’Oghuz extérieur et la mort de Beyrek}

Ce récit raconte un conflit intérieur d'une grande portée. Selon la coutume, Qazan organise chaque année une réception lors de laquelle il laisse ses vaisseaux fidèles piller sa maison. Cette année, il n'invite pas les seigneurs de la branche intérieure d'Oghuz et se contente de recevoir la branche extérieure. Ce favoritisme humiliant amène la branche intérieure à se rebeller. Beyrek est un seigneur qui refuse de trahir son seigneur par se joindre à cette révolte. Il se fait alors tuer en martyr. Qazan le venge et exécute son meurtrier avant de pardonner aux chefs révoltés et d'instaurer la paix. Ce récit se distingue des autres par le fait qu'il ne s'agit pas d'évoquer les hauts faits d'un jeune brave qui devient bey, mais le sacrifice d'un bey dont les hauts faits qui lui ont valu titre du bey ont déjà été racontés dans le troisième récit.

\section{Le récit où Qazan abat le dragon}

Qazan qui est surnommé " l'ancre de l'Azerbaïdjan » quitte ses hommes pour aller à la chasse tout seul. Sur son chemin il croise un dragon qui souffle du feu. Son père spirituel qui l'accompagne n'ose pas le dissuader de battre le dragon, mais lui explique seulement que «ce qu'on appelle dragon n'est qu'un serpent. » Grâce à un miracle, Qazan tue le dragon et se vêt de sa fourrure. Au début, les gens d'Oguz croient qu'il s'est transformé en dragon, mais ils finissent par le reconnaitre. Ce récit contient des thèmes classiques comme la chasse, le combat contre un animal monstrueux et la métamorphose. 


\section{Quatorzième récit}

Il s'agit d'un passage où Qazan raconte sa victoire dans une bataille contre les mécréants dans les territoires qui se trouvent aujourd'hui en Azerbaïdjan iranien.

\section{Annexe 2: cadre synthétique}

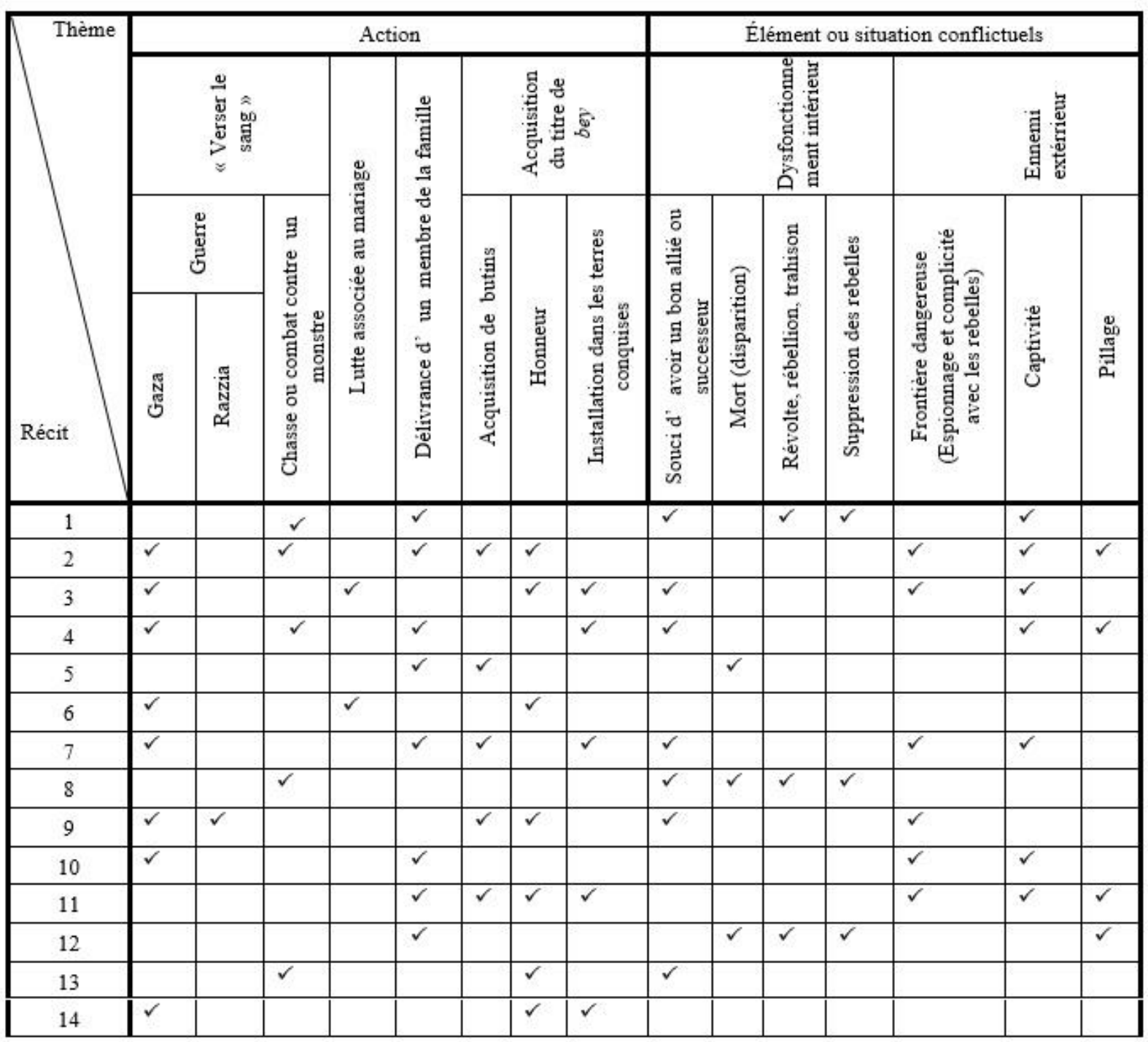

